\title{
Action discrimination: impact of apraxia
}

Mariella Pazzaglia ${ }^{1,2}$

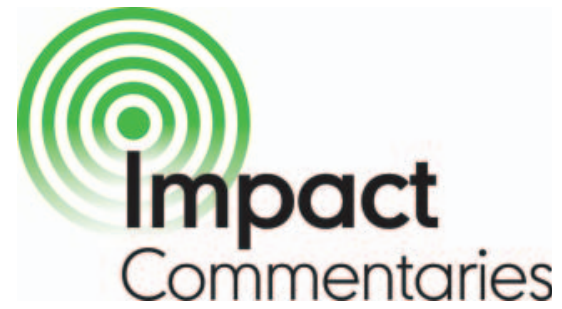

Mariella Pazzaglia from the IRCCS Fondazione Santa Lucia and University of Rome 'La Sapienza', in Rome describes a rapidly growing body of clinical research on the complex interplay of both production and comprehension mechanisms in actions derived from gesture comprehension studies described in patients with apraxia

The inextricable link between action perception and action execution was reported for the first time in a neuropsychological study of apraxia patients ${ }^{1}$ published 27 years ago in this journal. In that article, Rothi et al not only provided the first direct evidence of the existence of a bidirectional relationship between action perception and motor activity, but also provided causative information on the neural structures involved in the visual mapping of actions in patients with brain damage. Using a non-verbal paradigm, the authors identified a clear association between a deficit in performing gestures and the ability to recognise the pantomime of gestures appropriately in left hemisphere lesioned patients with apraxia but not in those with aphasia. These observations suggest that the representational aspects of gestures prevent action imitation and influence the execution of a given movement, typically affected in apraxia. Patients with apraxia presented lesions centred, mainly on the parietal cortex, but extending into the frontal regions. The authors revealed that, although both frontal and parietal structures are involved in the execution of actions, the left posterior regions seem to

\footnotetext{
'Department of Psychology, University of Rome 'La Sapienza', Rome, Italy; ${ }^{2}$ RCCCS Fondazione Santa Lucia, Rome, Italy

Correspondence to Dr M Pazzaglia, Department of Psychology, University of Rome 'La Sapienza', Via dei Marsi 78, Rome 00185, Italy; mariella.pazzaglia@uniroma1.it
}

\author{
PANTOMIME COMPREHENSION AND \\ IDEOMOTOR APRAXIA ${ }^{1}$ \\ Authors: Rothi L J, Heilman K M, \\ Watson R T \\ Published: 1985;48:207-10.
}

be primarily linked to the ability to specifically comprehend the meaning of pantomime.

This anatomical and functional investigation of apraxia patients highlighted, for the first time, a series of unexpected characteristics of these sensorimotor areas.

In fact, a decade later, neurophysiological research performed in monkeys showed that these two areas, which likely host mirror neurons, are the core regions of the system of action observation and execution. $^{2-5}$ In particular, it has been claimed that direct matching between action perception and execution is enabled by neural activity that overlaps largely in the inferior frontal gyrus and the inferior parietal lobe. ${ }^{6}$ One interpretation of such data is that motor system modulation during action perception facilitates and assists the reading of the actions of others and promotes readiness to predict movements and perform actions. ${ }^{67}$

The differential impact of parietal and frontal lesions on the recognition of gestures depicted by Rothi et al is well supported by clinical advances afforded by further research on apraxia.

Although lesions in the premotor cortex lead to impairment in judging the errors of the immediate purpose of an action performed by a model, ${ }^{6}$ it has been suggested that patients with lesions that affect the parietal lobe fail to recognise the spatial motor and kinematic components of actions. ${ }^{7}$ The precise neuroanatomical substrates involved in gesture discrimination in patients with apraxia remain unclear. ${ }^{8}$ In accordance with hierarchical modelling, via which an observed action can be 'understood' on the basis of simulation mechanisms, 9 action discrimination deficits (similar to action executions) in patients with apraxia can be described on multiple levels involving a complex neural network rather than a single structure. Therefore, although the parietal node may be more important for action simulation under conditions involving major kinematics and proprioceptive changes, the frontal lobe seems to be associated with the appropriateness of action representation.

It has also been indicated that left lateralised lesions in the frontal and parietal regions, both of which are essential for the visual discrimination of actions, might be associated with deficits in action production and recognition of sounds that are specifically linked to human actions. ${ }^{10}$ The inability to match sounds heard with images of hand and mouth related actions may also depend on tapped resources of the visual and mental representation of actions. Although visuomotor transfer impairments seem to reflect a more common form of disturbance in patients with apraxia, audiomotor deficits might also suggest the existence of more general deficits within the context of the multimodal representation of actions, regardless of whether they are mediated via visual or auditory means. ${ }^{11} 12$

Finally, and most importantly, we now know that patients with parietal damage and impaired ability to imitate or discriminate an observed action lose the capacity to monitor early phases of planning of their own movements. ${ }^{13}$ Apraxia patients with injury in parietal areas not only have major problems in comprehending actions but also frequently exhibit failure of the anticipatory motor process that drives forthcoming movements via predictive mechanisms. ${ }^{14}$

Inspired by the seminal study reported by Rothi et al, several studies have revealed a picture of apraxia that, although probably still incomplete, appears to be quite promising with regards to insights into the neural mechanisms that underlie perceptual motor code actions and an effective neurorehabilitation perspective. Based on the aspects of impairment described three decades ago, bidirectional training on the perceptual and motor codes has been developed to treat limb apraxia ${ }^{15}{ }^{16}$; this approach is recommended as being essential for the treatment of this type of disturbance. ${ }^{17}$ The relevance of the perceptual-motor coding identified in apraxia has encouraged the advancement of novel and effective treatments to cure the deficits associated with this disorder, and continues to be a valuable approach for gathering conclusive evidence on the role of the motor system in perception and cognition. 


\section{Competing interests None.}

Provenance and peer review Not commissioned; not externally peer reviewed.

To cite Pazzaglia M. I Neurol Neurosurg Psychiatry 2013;84:477-478.

Published Online First 5 March 2013

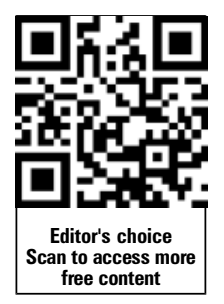

J Neurol Neurosurg Psychiatry 2013;84:477-478. doi:10.1136/jnnp-2012-304817

\section{REFERENCES}

1 Rothi LJ, Heilman KM, Watson RT. Pantomime comprehension and ideomotor apraxia. J Neurol Neurosurg Psychiatry 1985;48:207-10.

2 Fogassi L, Ferrari PF, Gesierich B, et al. Parietal lobe: from action organization to intention understanding. Science 2005;308:662-7.

3 Gallese V, Fadiga L, Fogassi L, et al. Action recognition in the premotor cortex. Brain 1996;119:593-609.

4 di Pellegrino G, Fadiga L, Fogassi L, et al. Understanding motor events: a neurophysiological study. Exp Brain Res 1992;91:176-80.

5 Grafton ST, Arbib MA, Fadiga L, et al. Localization of grasp representations in humans by positron emission tomography. 2. Observation compared with imagination. Exp Brain Res 1996;112:103-11.

6 Pazzaglia M, Smania N, Corato E, et al. Neura underpinnings of gesture discrimination in patients with limb apraxia. J Neurosci 2008:28:3030-41.

7 Kalenine S, Buxbaum LJ, Coslett HB. Critical brain regions for action recognition: lesion symptom mapping in left hemisphere stroke. Brain 2010;133:3269-80.

8 Hickok G. Eight problems for the mirror neuron theory of action understanding in monkeys and humans. J Cogn Neurosci 2009:21: 1229-43.

9 Kilner JM. More than one pathway to action understanding. Trends Cogn Sci 2011;15:352-7.

10 Pazzaglia M, Pizzamiglio L, Pes E, et al. The sound of actions in apraxia. Curr Biol 2008;18:1766-72.

11 Aglioti SM, Pazzaglia M. Representing actions through their sound. Exp Brain Res 2010;206:141-51.

12 Aglioti SM, Pazzaglia M. Sounds and scents in (social) action. Trends Cogn Sci 2011;15:47-55.

13 Fontana AP, Kilner JM, Rodrigues EC, et al. Role of the parietal cortex in predicting incoming actions. Neurolmage 2012:59:556-64.

14 Pazzaglia M. Does what you hear predict what you will do and say? Behav Brain Sci in press.

15 Smania N, Aglioti SM, Girardi F, et al. Rehabilitation of limb apraxia improves daily life activities in patients with stroke. Neurology 2006;67:2050-2.

16 Smania N, Girardi F, Domenicali C, et al. The rehabilitation of limb apraxia: a study in left-brain-damaged patients. Arch Phys Med Rehabil 2000;81:379-88.

17 Dovern A, Fink GR, Weiss PH. Diagnosis and treatment of upper limb apraxia. J Neurol 2012;259:1269-83. 